\title{
SYMMETRY ANALYSIS OF THE NUMERICAL INSTABILITIES IN THE TRANSFER MATRIX METHOD
}

\author{
Jose Miguel Luque-Raigon ${ }^{1,2 *}$, Janne Halme ${ }^{1}$, Hernan Miguez $^{2}$ and Gabriel Lozano ${ }^{2}$ \\ ${ }^{1}$ Department of Applied Physics, Aalto University School of Science, P.O. Box 15100, FI-00076 Espoo, Finland. \\ ${ }^{2}$ Instituto de Ciencia de Materiales de Sevilla (Consejo Superior de Investigaciones Científicas - Universidad de \\ Sevilla), C/Américo Vespucio 49, 41092 Sevilla, Spain.
}

"Corresponding Author. E-mail: jose.luque.raigon@aalto.fi

\begin{abstract}
This paper discusses the numerical exponential instability of the transfer matrix method (TMM) in the framework of the symmetry formalism. This numerical weakness is attributed to a series of increasingly extreme exponentials that appear in the TMM when it is applied to geometries involving total internal reflection (TIR) or very high absorption. We design a TMM formalism that identifies the internal symmetries of the multilayer geometry. These symmetries suggest particular transformations of reference system in the TMM that improve its ill-conditioned exponentials. To illustrate the numerical improvements, we present examples with calculations of electric fields.
\end{abstract}

Keywords: analytical solution, numerical instability, internal symmetries, absorption, total internal reflection, transfer matrix method.

PACS: 78.20.Bh; 78.20.Ci; 42.25.Bs 


\section{Introduction}

The solution to the Maxwell's equations associated with an interactive electromagnetic system can be reached with several mathematical methods that represent different approximations to the same solution. Yet, one method can be better than the other in terms of analytical structure, numerical stability and computer time. These differences are fundamental for obtaining efficient calculations in cases that are particularly susceptible to numerical errors [1,2].

The Transfer Matrix Method (TMM) is one of the most frequently used methods to understand the propagation of electromagnetic waves in one-dimensional multilayer structure. It is a very simple, accurate and fast method where a dielectric multilayer structure is decomposed to more easily solvable finite parts. TMM is a propagation method in the sense that it takes into account the transfer functions for one position to obtain the transfer function in other position of the structure. This procedure is iteratively repeated until all the different points of the structure are connected in a specific form, which is represented by the solution. However, the propagation of the electromagnetic waves in a multilayer is not one-directional because in each interface where refractive index changes, there is not only transmission but also reflection. Therefore, the set of interfaces generates multiple reflections along the structure which generates both forward and backward exponential waves in each layer (see chap. 3, ref. [3]). In this sense, the propagation in each point of the onedimensional multilayer is the result of an interference process between transmitted and reflected waves which come from the whole structure.

The well known issue of the TMM is that it can show numerical instabilities under certain extreme conditions concerning to the forward decreasing and backward increasing exponential waves. These exponential instabilities arise from the inversion of the propagation matrices appearing in the TMM. The numerical instabilities can be classified by the particular parameter in the exponential waves which generates them: a) instability associated with evanescent fields related to total internal reflection (TIR) [1], which comes from the imaginary component of the angle, and $b$ ) instability associated with attenuated fields due to absorption in layers that have high thickness/wavelength ratio [2], which comes from the imaginary 
component of the refractive index. The combination of these two contributions increases the chance and level of instability [1].

From the numerical point of view, the origin of these instabilities is the accumulation of scales (scalar real numbers with an absolute value higher than one) associated with two exponential terms in the TMM: one related to the forward propagation - a decreasing exponential term - and other related to the backward propagation - an increasing exponential term. These two different orders of magnitude in the TMM can produce round off inaccuracy in the calculations due to limited arithmetic precision of the computer. When calculations exceed the arithmetic precision an underflow instability occurs due to very small numbers and an overflow instability due to very large numbers [4].

To improve the numerical behaviour of the TMM, more stable methods have been developed, like TMM algorithms that use normalization and factorization of transfer matrix to avoid the instabilities from the exponentials terms [1]. There are also methods that expand the interaction problem in a non-exponential basis to avoid the problem [5]. One important method is the Scattering Matrix Method (SMM) [2, 5] which resolves the exponential instabilities by separating the forward-decreasing and backward-increasing exponentials and, as a consequence, shows a far greater region of stability than the usual TMM [6]. The $S$ matrix recursive algorithm is a SMM where exponential overflow is avoided by simply deleting the increasing exponential terms from the analytical solution [2]. Some relatively recent methods have successfully improved the TMM stability, like the enhanced transmittance matrix approach [1] and an analytically modified 2x2 TMM [7]. Numerical methods for particular cases of 1- and 2-dimensional photonic crystals (periodic structures) at different specific conditions have been developed by different groups [8-11].

This paper aims to clarify the theoretical foundation that allows obtaining equivalent solutions of the Maxwell's equations with TMM by means of reference system transformations in one dimension. We identify the internal symmetry of the TMM as a subjacent symmetry that arises from the electromagnetic homogeneity-isotropy inside each domain of the structure. This internal symmetry induces different TMMs configurations: those considering internal symmetry and those that do not consider it. The aim of the paper is 
to demonstrate theoretically how the solutions that consider the internal symmetries of the TMM improve the numerical stability and provide a simpler analytical structure than solutions that do not consider these symmetries. The numerical improvements are illustrated through simulations of a total internal reflection (TIR) multilayer that works either with an optically thin or a thick layer. The main contribution of this paper is that an improved TMM solution, which has been proposed in the literature [12] is explained here in detail through a novel symmetry based insight.

\section{Theoretical development}

Classical propagation and interaction of electromagnetic waves with a physical medium is described by the Maxwell's equations with a set of boundary conditions defined by the geometry of the interaction system. The interaction system consists of a finite non-periodic one-dimensional dielectric stack, called hereafter multilayer, the external media surrounding the multilayer, and the electromagnetic waves interacting with the former. The multilayer is infinitely extended through the $x$-axis and stratified along the $y$-axis direction in $n$ layers, which are indexed as $j=1,2, \ldots, n$ (see Fig. 1). Each $j$ layer is characterized by the thickness $d_{j}$, the complex electric permittivity $\tilde{\varepsilon}_{j}=\tilde{\varepsilon}_{j}(\omega)$ and the complex magnetic permeability $\tilde{\mu}_{j}=\tilde{\mu}_{j}(\omega)$. The $n$ layers are sandwiched between two semi-infinite non-absorptive external media, indexed as $j=0, n+1$, and characterized by the electromagnetic properties $\tilde{\varepsilon}_{0}=\tilde{\varepsilon}_{0}(\omega), \quad \tilde{\mu}_{0}=\tilde{\mu}_{0}(\omega)$ for the incoming propagation medium, and $\tilde{\varepsilon}_{n+1}=\tilde{\varepsilon}_{n+1}(\omega), \quad \tilde{\mu}_{n+1}=\tilde{\mu}_{n+1}(\omega)$ for the outgoing propagation medium (see Fig. 1). The electromagnetic properties of the domains, i.e. the layers and the external media, are given by complex frequency-dependent scalar refractive indices $\tilde{n}_{j}^{2}(\omega)=\tilde{\varepsilon}_{j}(\omega) \tilde{\mu}_{j}(\omega)$, the real part of which describes dispersion and the imaginary part absorption. The electromagnetic properties define each domain in a homogeneous, isotropic and linear manner. The homogeneity and isotropy indicate that electromagnetic properties are scalar numbers, i.e. invariant under translations and rotations inside each domain. The linearity 
of each domain allows using matrix methods to characterize the interaction system, like the Transfer Matrix Method (TMM).

The complex electromagnetic fields $\tilde{\mathbf{E}}_{j}(\mathbf{r}), \tilde{\mathbf{H}}_{j}(\mathbf{r})$ are described in a vectorial form using the framework of the classical electrodynamics. The complex angle $\tilde{\theta}_{j}$ between the complex wavevector direction $\tilde{\mathbf{k}}_{j}$ and the stratified unitary direction vector $\hat{\mathbf{y}}$ of the multilayer is taken into account as a variable; therefore, $\tilde{\beta}_{j}, \tilde{\alpha}$ are the complex wavevector projections along $y$-axis and $x$-axis respectively, which are given by

$$
\tilde{\mathbf{k}}_{j}=\tilde{\beta} \hat{\beta}_{j} \hat{\mathbf{y}}+\tilde{\alpha} \hat{\mathbf{x}} \equiv \frac{\omega}{c} \tilde{n}_{j}(\omega)\left\{\cos \left(\tilde{\theta}_{j}\right) \hat{\mathbf{y}}+\sin \left(\tilde{\theta}_{j}\right) \hat{\mathbf{x}}\right\} \equiv \frac{2 \pi}{\lambda} \tilde{n}_{j}(\lambda)\left\{\cos \left(\tilde{\theta}_{j}\right) \hat{\mathbf{y}}+\sin \left(\tilde{\theta}_{j}\right) \hat{\mathbf{x}}\right\}
$$

where $j=0,1, \ldots, n, n+1$ (see Fig. 2). The Maxwell's equations are defined without free charges inside the multilayer and their solution is considered in a time-independent approximation by using harmonic stationary modes. Furthermore, a positive convention for the field time-phase is considered, which gives the solution as

$$
\tilde{\mathbf{E}}_{j}(\mathbf{r}, t)=\tilde{\mathbf{E}}_{j}(\mathbf{r}) e^{i \omega t} ; \quad \tilde{\mathbf{H}}_{j}(\mathbf{r}, t)=\tilde{\mathbf{H}}_{j}(\mathbf{r}) e^{i \omega t}
$$

for the electric field $\tilde{\mathbf{E}}_{j}(\mathbf{r}, t)$ and magnetic field $\tilde{\mathbf{H}}_{j}(\mathbf{r}, t)$. Furthermore, following the standard convention, the field vectors are expressed in the basis of two vector components corresponding to the two polarization modes: $s$-polarization or transversal electric mode TE, and $p$-polarization or transversal magnetic mode TM (see Fig. 2). These polarization components are represented by $\left\{\tilde{\mathbf{E}}_{j}^{s}(\mathbf{r}), \tilde{\mathbf{H}}_{j}^{s}(\mathbf{r}) ; \tilde{\mathbf{k}}_{j}\right\}$ and $\left\{\tilde{\mathbf{E}}_{j}^{p}(\mathbf{r}), \tilde{\mathbf{H}}_{j}^{p}(\mathbf{r}) ; \tilde{\mathbf{k}}_{j}\right\}$, where each components are completely built up from the Maxwell's equations together with the electric fields associated to each mode $\tilde{\mathbf{E}}_{j}^{s}(\mathbf{r}), \tilde{\mathbf{E}}_{j}^{p}(\mathbf{r})$ (see chap. 3, ref. [3]). 
In the following three sections we develop the theoretical analysis of the TMM symmetry. First, we summarize the solutions that do not consider these symmetries, but written in a new TMM formalism more adequate to detect the internal symmetries of the multilayer geometry. Second, we discuss the origin of the numerical instabilities in TMM in the framework of these solutions. Third, we show how transformations of reference system considering (preserving) the symmetries improve the numerical stability and simplify the analytical solution.

\section{Solutions that do not consider the internal symmetries of the TMM}

In this section we define a new formalism adequate for analyzing in detail the geometrical structure of the TMM by introducing a new concept: the partial propagation matrix. This matrix differs from the propagation matrix that appears in the literature [12], in the sense that the propagation matrix is defined for a complete domain, whereas the partial propagation matrix is defined associated to one part of the domain, namely the part between the origin of that domain and a point inside the same domain. Therefore, the geometrical spatial structure of the TMM is characterized by a reference system with its origin. Each point of the multilayer is described in this reference system by associating a partial propagation matrix to this point. This new matrix concept allows understanding the TMM iteration at the point-by-point level. Hence, the partial propagation matrix in one point is obtained from the partial propagation matrix in any other point by iterations that are propagated in a continuous or discrete form. In this work, we use this matrix assignment to develop a solution in the case of a one-dimensional structure, in order to provide a clear representation of the symmetry method.

The solutions of the Maxwell's equation that do not consider the internal symmetries of TMM, are associated to a single arbitrary reference system $\left\{y ; y_{0}\right\}$ along $y$-axis and an arbitrary origin $y=y_{0}$ for all the domains (see Fig. 3). These solutions are expressed in the new formalism by 


$$
\begin{aligned}
& \int\left(e^{-i \tilde{\beta}_{0}\left(y-y_{0}\right)}+\tilde{E}_{0}^{\prime-} e^{+i \tilde{\beta}_{0}\left(y-y_{0}\right)}\right) e^{-i \tilde{\alpha} x}=\left(\begin{array}{ll}
1 & 1
\end{array}\right)\left(\mathbf{P}_{0}^{-1}\right)_{y-y_{0}}\left(\begin{array}{c}
1 \\
\tilde{E}_{0}^{\prime-}
\end{array}\right) e^{-i \tilde{\alpha} x} \\
& \text { with } \mathrm{y} \in\left(-\infty, y_{0}\right) \equiv 0 \text {-region; } x \in\left(x_{0},+\infty\right)
\end{aligned}
$$

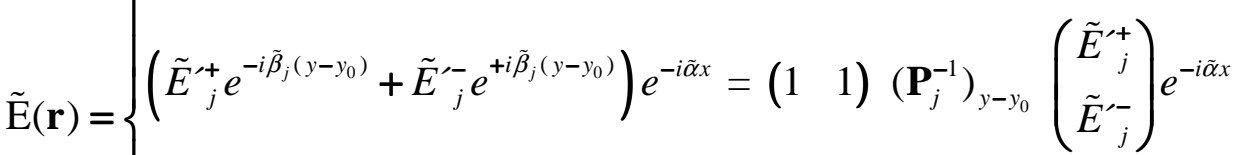

$$
\begin{aligned}
& \text { with } \mathrm{y} \in\left(y_{j-1}, y_{j}\right) \equiv j \text {-region; } x \in\left(x_{0},+\infty\right) \\
& \tilde{E}_{n+1}^{\prime+} e^{-i \tilde{\beta}_{n+1}\left(y-y_{0}\right)} e^{-i \tilde{\alpha} x}=\left(\begin{array}{ll}
1 & 1
\end{array}\right)\left(\mathbf{P}_{n+1}^{-1}\right)_{y-y_{0}}\left(\begin{array}{c}
\tilde{E}_{n+1}^{\prime+} \\
0
\end{array}\right) e^{-i \tilde{\alpha} x} \\
& \text { with } \mathrm{y} \in\left(y_{n},+\infty\right) \equiv(n+1) \text {-region; } x \in\left(x_{0},+\infty\right)
\end{aligned}
$$

where $\left(\mathbf{P}_{0}\right)_{y-y_{0}} \equiv\left(\begin{array}{cc}e^{i \tilde{\beta}_{0}\left(y-y_{0}\right)} & 0 \\ 0 & e^{-i \tilde{\beta}_{0}\left(y-y_{0}\right)}\end{array}\right) ; \quad\left(\mathbf{P}_{j}\right)_{\left(y-y_{0}\right)} \equiv\left(\begin{array}{cc}e^{i \tilde{\beta}_{j}\left(y-y_{0}\right)} & 0 \\ 0 & e^{-i \tilde{\beta}_{j}\left(y-y_{0}\right)}\end{array}\right)$

where $j=1, \ldots, n$. The multiple reflection in each layer are taken into account through the forward and backward wave amplitudes $\tilde{E}_{j}^{\prime+}$ and $\tilde{E}_{j}^{\prime-}$ respectively. The semi-infinite external media satisfy conditions $y_{-1}=-\infty ; \quad y_{n+1}=+\infty$, as well as $\tilde{E}_{n+1}^{\prime-}=0$, which means that there are no reflected waves in the outgoing medium, and $\tilde{E}_{0}^{\prime+}=1$, which means that all the incident waves are normalized to unity. We have defined in (3) the partial propagation matrices $\left(\mathbf{P}_{j}\right)_{y-y_{0}}$, where $j=0,1, \ldots, n$, that characterize the electromagnetic behaviour in the $y$-point of the $j$-domain with respect to the origin $y_{0}$. Note that each solution (3) has a reference system $\left\{y ; y_{0}\right\}$ characterized with the same origin $y_{0}$ for all the domains (Fig. 3).

The field amplitudes $\tilde{E}_{j}^{\prime+}, \tilde{E}_{j}^{\prime-}$ in (3) are obtained from the initial field amplitudes $\tilde{E}_{n+1}^{\prime-}=0$ and $\tilde{E}_{0}^{\prime+}=1$ by means of a recursive rule that links them between neighboring domains via the interface boundary conditions. These boundary conditions are equivalent to the integral form of Maxwell's equations at all the interfaces $y=y_{j}$, where $j=0,1, \ldots, n-1, n$. The boundary conditions establish a connection for 
the field amplitudes associated to consecutive domains just at the interface between both. When those relations are recursively iterated from the initial conditions $\tilde{E}_{n+1}^{\prime-}=0$ and $\tilde{E}_{0}^{\prime+}=1$, then all the rest of the field amplitudes $\tilde{E}_{j}^{\prime+}, \tilde{E}_{j}^{\prime-}$ are obtained. The recursive method is called the Transfer Matrix Method (TMM), and it can be propagated in the increasing or decreasing index-direction. The recursive rules that characterizes the propagation of the TMM in the increasing index-direction, are given by

$\left(\mathbf{P}_{j}\right)_{y_{0}}\left(\begin{array}{c}\tilde{E}^{\prime+} \\ \tilde{E}^{\prime-}\end{array}\right)=\left[\left(\mathbf{P}_{j}\right)_{y_{j-1}} \mathbf{D}_{j}^{-1} \mathbf{D}_{j-1}\left(\mathbf{P}_{j-1}\right)_{y_{j-1}^{-1}}^{-1}\right]\left(\mathbf{P}_{j-1}\right)_{y_{0}}\left(\begin{array}{c}\tilde{E}_{j-1}^{\prime+} \\ \tilde{E}^{\prime-1}\end{array}\right)$

where $\left(\mathbf{P}_{j}\right)_{y_{k}} \equiv\left(\begin{array}{cc}e^{i \tilde{\beta}_{j} y_{k}} & 0 \\ 0 & e^{-i \tilde{\beta}_{j} y_{k}}\end{array}\right) ; \quad \mathbf{D}_{j} \equiv\left\{\begin{array}{cc}\left(\begin{array}{cc}1 & 1 \\ \tilde{n}_{j} \cos \left(\tilde{\theta}_{j}\right) & -\tilde{n}_{j} \cos \left(\tilde{\theta}_{j}\right)\end{array}\right) & s \text {-polarization } \\ \left(\begin{array}{cc}\cos \left(\tilde{\theta}_{j}\right) & \cos \left(\tilde{\theta}_{j}\right) \\ \tilde{n}_{j} & -\tilde{n}_{j}\end{array}\right) & p \text {-polarization }\end{array}\right.$

with $j=1, \ldots, n+1$, and reference system origin in $y_{0}$ for all the regions

where the forward and backward wave propagation along the multilayer is factored in two kind of matrices: the dynamical matrices and the propagation matrices. The dynamical matrices $\mathbf{D}_{j}$ and $\mathbf{D}_{j-1}$ are associated to the transformation of field amplitudes across the interface $j-1$, that is between domains $j-1$ and $j$, by the operator $\mathbf{D}_{j}^{-1} \mathbf{D}_{j-1}$. The TMM (4) has the components $\left(\mathbf{P}_{j-1}\right)_{y_{j-1}}\left(\mathbf{P}_{j-1}\right)_{y_{0}}^{-1} \equiv\left(\mathbf{P}_{j-1}\right)_{y_{j-1}-y_{0}}$ that correspond to partial propagation matrices at the point $y_{j-1}$ of $(j-1)$ - domain, with respect to a common external origin $y=y_{0}$ (Fig. 3). The dynamical matrices for each interface separate the wave propagation in $s$-polarization and $p$-polarization waves, as described in equation (4). Therefore, it is necessary to introduce the notation $\tilde{E}_{j}^{s+}, \tilde{E}_{j}^{\prime p+}$ for the complex field amplitudes of the $s$ and $p$ modes in the forward propagating wave, and $\tilde{E}_{j}^{\prime s-}, \tilde{E}_{j}^{\prime p-}$ for the complex field amplitudes of the $s$ and $p$ modes in the backward propagating wave. Note 
that the exponent signs in these forward-backward propagating waves are established by the positive convention for the fields time-phases given in equation (2).

The finiteness conditions for the spatial propagation of the fields $\tilde{\mathbf{E}}_{j}^{\prime s}(\mathbf{r}), \tilde{\mathbf{E}}_{j}^{\prime p}(\mathbf{r})$ impose that the limits $\lim _{y \rightarrow+\infty} \tilde{E}_{j}^{s+}, \tilde{E}_{j}^{\prime p+}, \lim _{y \rightarrow-\infty} \tilde{E}_{j}^{\prime s-}, \tilde{E}_{j}^{\prime p-}$ and $\lim _{x \rightarrow+\infty} \tilde{E}_{j}^{\prime s+}, \tilde{E}_{j}^{\prime p+}, \tilde{E}_{j}^{\prime s-}, \tilde{E}_{j}^{\prime p-}$ are finite numbers, and they are fulfilled by the wavevector conditions

$$
\operatorname{Im}\left(\tilde{\beta}_{j}\right) \leq 0, \quad \operatorname{Im}(\tilde{\alpha}) \leq 0 ; \quad \text { where } j=0,1, \ldots, n, n+1
$$

where $\operatorname{Im}(\cdot)$ is the imaginary part of the corresponding wavevector projection. These conditions must be fulfilled for the propagation waves in all domains and interfaces - that is, all position space; only in this manner the analytical infinite-instability is avoided from the spatial exponential functions. The set of equations (3)-(4)-(5), together with single arbitrary reference system $\left\{y ; y_{0}\right\}$ for all the homogeneous domains, constitutes an analytical solution of the Maxwell's equations that does not consider the internal symmetries of the TMM. The solution is invariant in form under arbitrary transformations of the reference system.

\section{Origin of the numerical instabilities in TMM}

The main problem of the TMM concerns to the numerical instabilities that come from the forward decreasing and backward increasing exponential waves. They originate from the imaginary component of the angle that is associated to total internal reflection (TIR) processes [1], as well as from the imaginary component of the refractive index that is associated to absorption in layers that have high thickness/wavelength ratio [2]. These parameters generate in the iterative TMM an accumulation of scales which can be close to (inaccuracies) or exceed (overflow) the arithmetic precision of the computer. 
In this section we intend to give a summarized explanation about the origin of the TMM instabilities by following the references $[1,2]$. We consider a solution (3)-(4)-(5) in a single reference system $\left\{y ; y_{0}\right\}$ with an arbitrary fixed origin in $y=y_{0}$ for all the domains (see Fig. 3). From the initial amplitude field conditions $\tilde{E}_{n+1}^{\prime-}=0$ and $\tilde{E}_{0}^{\prime+}=1$, the iteration of the recursive rule for the TMM (4) provides the field amplitudes for all the domains $\tilde{E}_{j}^{\prime+}, \tilde{E}_{j}^{\prime-}$. This TMM propagation along the whole thickness of the multilayer (not be confused with the waves propagation) results in accumulation of the scale through the exponential components of (4) due to the recursive matrix multiplications. In this situation, if the wavevector projection $\tilde{\beta}_{j}$ is a pure real number, such as in the cases of non-absorptive structures and non-TIR processes, the exponential corresponds to a pure oscillation with a maximum value of 1 normalized to by the initial condition $\tilde{E}_{0}^{\prime+}=1$ and numerical problems do not exist. However, if the wavevector has an imaginary component, such as in the cases of absorptive structures or TIR processes, the oscillatory part appears multiplied by exponentially increasing and decreasing terms (scale terms) with a maximum numerical value that could be so very high to generate numerical instabilities. It can therefore be concludes that the numerical instabilities originate from a non-zero imaginary part in the $y$-wavevector. The scales and oscillations appearing in the partial propagation matrices $\left(\mathbf{P}_{j}\right)_{y_{j}-y_{0}}$ of the TMM are represented as follows

$$
\begin{gathered}
\left(\mathbf{P}_{j}\right)_{y_{j}-y_{0}} \equiv\left(\begin{array}{cc}
e^{-\operatorname{Im} \tilde{\beta}_{j}\left(y_{j}-y_{0}\right)} e^{i R e \tilde{\beta}_{j}\left(y_{j}-y_{0}\right)} & 0 \\
0 & e^{+\operatorname{Im} \tilde{\beta}_{j}\left(y_{j}-y_{0}\right)} e^{-i R e \tilde{\beta}_{j}\left(y_{j}-y_{0}\right)}
\end{array}\right) ; \\
\text { where } e^{\mp \operatorname{Im} \tilde{\beta}_{j}\left(y_{j}-y_{0}\right)}=\mathrm{e}^{\mp 2 \pi \frac{\left(y_{j}-y_{0}\right)}{\lambda}\left\{\operatorname{Im}\left[\tilde{n}_{j}(\lambda)\right] \operatorname{Re}\left[\cos \left(\tilde{\theta}_{j}\right)\right]+\operatorname{Re}\left[\tilde{n}_{j}(\lambda)\right] \operatorname{Im}\left[\cos \left(\tilde{\theta}_{j}\right)\right]\right\}} \\
e^{ \pm i R e \tilde{\beta}_{j}\left(y_{j}-y_{0}\right)}=\mathrm{e}^{ \pm i 2 \pi \frac{\left(y_{j}-y_{0}\right)}{\lambda}\left\{\operatorname{Re}\left[\tilde{n}_{j}(\lambda)\right] \operatorname{Re}\left[\cos \left(\tilde{\theta}_{j}\right)\right]-\operatorname{Im}\left[\tilde{n}_{j}(\lambda)\right] \operatorname{Im}\left[\cos \left(\tilde{\theta}_{j}\right)\right]\right\}}
\end{gathered}
$$

where $\operatorname{Re}(\cdot)$ are the real part of the argument, $e^{\mp I m \tilde{\beta}_{j}\left(y_{j}-y_{0}\right)}$ the scales and $e^{ \pm i R e \tilde{\beta}_{j}\left(y_{j}-y_{0}\right)}$ the oscillations. 
We know that the imaginaries parts $\operatorname{Im}\left[\tilde{n}_{j}(\lambda)\right], \operatorname{Im}\left[\cos \left(\tilde{\theta}_{j}\right)\right]$ can be zero or non-zero; the non-zero imaginary part gives origin to the exponential decreases and increases. In relation (6), we can understand that the exponential decrease and increase $e^{\mp \operatorname{Im} \tilde{\beta}_{j}\left(y_{j}-y_{0}\right)}$ are originated from a non-null imaginary part of the angle $\operatorname{Im}\left[\cos \left(\tilde{\theta}_{j}\right)\right]$ and/or a non-null imaginary part of refractive index $\operatorname{Im}\left[\tilde{n}_{j}(\lambda)\right]$, and are enhanced by both real parts $\operatorname{Re}\left[\tilde{n}_{j}(\lambda)\right], \operatorname{Re}\left[\cos \left(\tilde{\theta}_{j}\right)\right]$ and the ratio of thickness (position coordinate) to the wavelength $\left(y_{j}-y_{0}\right) / \lambda$

The physical-geometrical processes that can produce numerical exponential instabilities in the TMM are the total internal reflection (TIR) from the imaginary component of the angle $\operatorname{Im}\left[\cos \left(\tilde{\theta}_{j}\right)\right]$, as well as absorption in layers from the imaginary component of the refractive index $\operatorname{Im}\left[\tilde{n}_{j}(\lambda)\right]$. If there appears one or both of these previous physical processes, then numerical instabilities can arise and be accumulated through a high geometrical thickness/wavelength ratio $\left(y_{j}-y_{0}\right) / \lambda$ (see relations in (6)). Hence, the exponential instabilities appear more frequently in interaction systems with absorption layers and/or TIR that, additionally, work at high thickness/wavelength ratio [1,2]. The scale accumulation, quantified in (6), can be improved by reference system transformations that consider the internal symmetries of the TMM.

\section{Solutions that consider the internal symmetries of the TMM}

The solution (3)-(4)-(5) in reference system $\left\{y ; y_{0}\right\}$ is invariant in form under transformations of reference system. Therefore, it is possible to get the different equivalent solutions by means of mappings of that solution associated to reference system transformations (see Fig. 3). These invariances are associated to the fact that there is neither a special reference system (translation) nor a fundamental length scale (dilatation) for the Maxwell's equations (see pp. 34-35, ref. [3]). The dilatational invariance is merely a classical electrodynamics property - from the physical point-of-view the quantum electrodynamics restricts the applicability of these considerations below the molecular and atomic length scales. 
Additionally, the solution (3)-(4)-(5) in reference system $\left\{y ; y_{0}\right\}$ has implicit internal symmetries, associated to the homogeneity-by-domain (domain-wise homogeneity) of the multilayer structure. These internal symmetries can be incorporated in the TMM by choosing an adequate reference system that simplifies the analytical structure and improves the numerical behaviour of the TMM.

The electromagnetic homogeneity of each domain in the structure is the origin of the internal symmetries of the TMM. Mathematically, the homogeneity can be stated as: if two arbitrary points $y_{1}, \mathrm{y}_{2}$ are localized inside the same homogeneous $u$-domain of the interaction system, then it follows that their electromagnetic behaviour through partial propagation matrices $\left(\mathbf{P}_{u}\right)_{y_{1}-y_{0}},\left(\mathbf{P}_{u}\right)_{y_{2}-y_{0}}$, referred to any reference system $\left\{y ; y_{0}\right\}$, commute $\left[\left(\mathbf{P}_{u}\right)_{y_{1}-y_{0}},\left(\mathbf{P}_{u}\right)_{y_{2}-y_{0}}\right]=\left(\mathbf{P}_{u}\right)_{y_{1}-y_{0}}\left(\mathbf{P}_{u}\right)_{y_{2}-y_{0}}-\left(\mathbf{P}_{u}\right)_{y_{2}-y_{0}}\left(\mathbf{P}_{u}\right)_{y_{1}-y_{0}}=0$. In this last sense, the partial propagation matrices are internal symmetries of the TMM which, consequently, can be used to build up the mappings. The management of the numerical exponential instability through these particular internal symmetries is the key in the present paper. They are defined as internal symmetries because they come from a symmetry that appears in a natural form inside the TMM structure.

We employ the solution (3)-(4)-(5) in reference system $\left\{y ; y_{0}\right\}$ to build up a new improved solution from the mapping associated to the reference system transformation (see Fig. 3), expressed analytically as

$$
\begin{array}{ll}
y-y_{0} \rightarrow y-y_{j}<0, & \text { for } j=0 \\
y-y_{0} \rightarrow y-y_{j-1}>0, & \text { for } j=1, \ldots, n, n+1 \quad \text { (rest of the regions) }
\end{array}
$$

which impose the fundamental conditions that if $y-y_{0} \in j$-region in the reference system of the solution (3)-(4)-(5), then $y-y_{j-1} \in j$-region in the new reference system (mapping each domain into itself), and each interface remains invariant (mapping each interface into itself). The physical reason why the transformation (7) makes explicit the internal symmetries of the TMM is based on the preference given to the interface positions in the new reference system, which are assigned as origins of their corresponding domains (see Fig. 3). The mathematical reasons will be more clearly explained at the end of this section. This 
transformation (7) corresponds to the composition of one translation that comes from the real part of the wavevector, with one dilatation that comes from the imaginary part of the wavevector, both quantified as the new origin values in $y=y_{j-1}$ for each $j$-region (see Fig. 3). The associated mapping between the solutions (see Fig. 3) are given by

$$
\begin{aligned}
& \left(\mathbf{P}_{0}\right)_{y_{0}}\left(\begin{array}{c}
\tilde{E}_{j}^{\prime+} \\
\tilde{E}_{j}^{\prime-}
\end{array}\right) \equiv\left(\mathbf{P}_{0}\right)_{y_{0}}\left(\begin{array}{c}
\tilde{E}_{j}^{+} \\
\tilde{E}_{j}^{-}
\end{array}\right) ; \quad \text { for incoming medium } j=0 \\
& \left(\mathbf{P}_{j}\right)_{y_{0}}\left(\begin{array}{c}
\tilde{E}_{j}^{\prime+} \\
\tilde{E}_{j}^{\prime-}
\end{array}\right) \equiv\left(\mathbf{P}_{j}\right)_{y_{j-1}}\left(\begin{array}{c}
\tilde{E}_{j}^{+} \\
\tilde{E}_{j}^{-}
\end{array}\right) ; \quad \text { for regions } j=1, \ldots, n, n+1
\end{aligned}
$$

where matrices $\left(\mathbf{P}_{j}\right)_{y_{k}}$ are defined in equations (3)-(4). The mapping (8) that comes from the reference system transformation (7) is right because equations (3)-(4)-(5) are invariant in form under an arbitrary transformation of the reference system, as already mentioned. From the operational point of view, mapping (8) is built up as the action of the internal symmetry operators $\left(\mathbf{P}_{0}\right)_{y-y_{0}},\left(\mathbf{P}_{j}\right)_{y-y_{0}},\left(\mathbf{P}_{j}\right)_{y-y_{j-1}}$ on the field amplitude vectors. Furthermore, note that the transformation of reference system (7) changes the amplitude coefficients through the relation (8). The final solution that considers the internal symmetries of the TMM is achieved by introducing the mapping (8) in the initial solution (3)-(4)-(5), which gives 


$$
\tilde{\mathrm{E}}(\mathbf{r})=\left\{\begin{array}{c}
\left(e^{-i \tilde{\beta}_{0}\left(y-y_{0}\right)}+\tilde{E}_{0}^{-} e^{+i \tilde{\beta}_{0}\left(y-y_{0}\right)}\right) e^{-i \tilde{\alpha} x}=\left(\begin{array}{ll}
1 & 1
\end{array}\right)\left(\mathbf{P}_{0}^{-1}\right)_{y-y_{0}}\left(\begin{array}{c}
1 \\
\tilde{E}_{0}^{-}
\end{array}\right) e^{-i \tilde{\alpha} x} \\
\text { with } \mathrm{y} \in\left(-\infty, y_{0}\right) \equiv \text {-region; } x \in\left(x_{0},+\infty\right) \\
\left(\tilde{E}_{j}^{+} e^{-i \tilde{\beta}_{j}\left(y-y_{j-1}\right)}+\tilde{E}_{j}^{-} e^{+i \tilde{\beta}_{j}\left(y-y_{j-1}\right)}\right) e^{-i \tilde{\alpha} x}=\left(\begin{array}{ll}
1 & 1
\end{array}\right)\left(\mathbf{P}_{j}^{-1}\right)_{y-y_{j-1}}\left(\begin{array}{c}
\tilde{E}_{j}^{+} \\
\tilde{E}_{j}^{-}
\end{array}\right) e^{-i \tilde{\alpha} x} \\
\text { with y } \in\left(y_{j-1}, y_{j}\right) \equiv j \text {-region; } x \in\left(x_{0},+\infty\right) \\
\tilde{E}_{n+1}^{+} e^{-i \tilde{\beta}_{n+1}\left(y-y_{n}\right)} e^{-i \tilde{\alpha} x}=\left(\begin{array}{ll}
1 & 1
\end{array}\right)\left(\mathbf{P}_{n+1}^{-1}\right)_{y-y_{n}}\left(\begin{array}{c}
\tilde{E}_{n+1}^{+} \\
0
\end{array}\right) e^{-i \tilde{\alpha} x} \\
\text { with y } \in\left(y_{n},+\infty\right) \equiv(n+1)-\text { region; } x \in\left(x_{0},+\infty\right)
\end{array}\right.
$$

where $j=1, \ldots, n$; the semi-infinite external media satisfying $y_{-1}=-\infty, y_{n+1}=+\infty, \tilde{E}_{n+1}^{-}=0$ and $\tilde{E}_{0}^{+}=1$. The matrix $\left(\mathbf{P}_{j}\right)_{y-y_{j-1}} \equiv\left(\mathbf{P}_{j}\right)_{y}\left(\mathbf{P}_{j}\right)_{y_{j-1}}^{-1}$ is the partial propagation matrix for the point $y$ in the $j$-domain with respect to the origin $y_{j-1}$. The recursive rules for transfer matrix method (TMM) that consider the internal symmetries can be obtained applying mapping (8) and multiplying by $\left(\mathbf{P}_{j}\right)_{y_{j-1}}^{-1}$ in both sides of the equation (4), with the following result

$$
\begin{aligned}
& \left(\begin{array}{c}
\tilde{E}_{1}^{+} \\
\tilde{E}_{1}^{-}
\end{array}\right)=\mathbf{D}_{1}^{-1} \mathbf{D}_{0}\left(\begin{array}{c}
1 \\
\tilde{E}_{0}^{-}
\end{array}\right) ; \quad\left(\begin{array}{c}
\tilde{E}_{j}^{+} \\
\tilde{E}_{j}^{-}
\end{array}\right)=\mathbf{D}_{j}^{-1} \mathbf{D}_{j-1} \mathbf{P}_{j-1}^{-1}\left(\begin{array}{c}
\tilde{E}_{j-1}^{+} \\
\tilde{E}_{j-1}^{-}
\end{array}\right) \quad \text { with } j=2, \ldots, n+1 \\
& \operatorname{being} \mathbf{P}_{j} \equiv\left(\mathbf{P}_{j}\right)_{y_{j}-y_{j-1}} \equiv\left(\mathbf{P}_{j}\right)_{y_{j}}\left(\mathbf{P}_{j}\right)_{y_{j-1}}^{-1}=\left(\begin{array}{cc}
e^{i \tilde{\beta}_{j}\left(y_{j}-y_{j-1}\right)} & 0 \\
0 & e^{-i \tilde{\beta}_{j}\left(y_{j}-y_{j-1}\right)}
\end{array}\right) \\
& \mathbf{D}_{j}=\left\{\begin{array}{cc}
\left(\begin{array}{cc}
1 & 1 \\
\tilde{n}_{j} \cos \left(\tilde{\theta}_{j}\right) & -\tilde{n}_{j} \cos \left(\tilde{\theta}_{j}\right)
\end{array}\right) & s \text {-polarization } \\
\left(\begin{array}{cc}
\cos \left(\tilde{\theta}_{j}\right) & \cos \left(\tilde{\theta}_{j}\right) \\
\tilde{n}_{j} & -\tilde{n}_{j}
\end{array}\right) & p \text {-polarization }
\end{array}\right.
\end{aligned}
$$


The complete solution of the Maxwell's equations that consider the internal symmetries of the TMM is provided by the equations (5)-(9)-(10) in a reference system that has one origin at the initial point of each domain defined as $\left\{\left(y ; y_{0}\right) \cup\left(y ; y_{1}-y_{0}\right) \cup \ldots \cup\left(y ; y_{n}-y_{n-1}\right)\right\}$ (see Fig. 3). The mathematical reason of the simplification of the iterative rule for the TMM (10) with respect to the iterative rule (4) is associated directly with the mapping (8) between both rules. The substitutions for that special mapping $\left(\mathbf{P}_{0}\right)_{y_{0}} \rightarrow\left(\mathbf{P}_{0}\right)_{y_{0}} ;\left(\mathbf{P}_{j}\right)_{y_{0}} \rightarrow\left(\mathbf{P}_{j}\right)_{y_{j-1}}$, where $j=1, \ldots, n+1$, render that each matrix $\left(\mathbf{P}_{j}\right)_{y_{0}}$ in the first member of the TMM (4) is exactly canceled with each matrix $\left(\mathbf{P}_{j}\right)_{y_{j-1}}$ in the second member of (4), for all domain $j=1, \ldots, n+1$. This fact corresponds to an analytical and numerical simplification of the TMM given by (5)-(9)-(10) with respect to the TMM given by (3)-(4)-(5). This simplified solution arises because the transformation (7) and its mapping (8) are fitted to the internal symmetries of the TMM.

The solution (5)-(9)-(10) is used in the reference [12], where it is proposed without any explanation about its analytical or numerical improvement with respect to other equivalent solutions. Here, we have shown that solution (5)-(9)-(10) represents analytically and numerically an improved mapping with respect to the solution (3)-(4)-(5), reachable by considering the internal symmetries of the TMM. The TMM rule defined by (10) is analytically simpler than the TMM rule defined in (4). Additionally, (10) shows lower values in the components of its propagation matrices than (4) which results to better numerical stability, as is demonstrated by simulations in the following section. The analytical structure and numerical behaviour for both two solutions are different because they are referred to different reference systems. Although the new mapping method proposed for the TMM is discussed here with regards to the specific case of a onedimensional optical multilayer, it is easy to envision that it would be applicable also to more complex twoand three-dimensional structures, taking into account their particular internal symmetries, and yielding similar analytical and numerical benefits as in the present one-dimensional case. 


\section{Calculations and results}

In this section, we illustrate through numerical calculations how the mapping obtained in the last section improves the numerical stability. We present one-to-one comparison of the model given by (3)-(4)-(5) to the improved model given by (5)-(9)-(10) under critical conditions that come from the imaginary parts of the refractive index and/or the angle. The situations calculated here are: a) total internal reflection with a very thin absorptive layer, and $b$ ) total internal reflection with a thick absorptive layer. The numerical calculations were performed with Matlab version 7.11.0.584 (R2010b) using a processor Intel Xeon CPU E3-1230 @ $3.20 \mathrm{GHz}$.

We employ a multilayer structure that shows total internal reflection (TIR) with either a very thin absorptive layer (first simulations) or a thick absorptive layer (second simulations), and perform angledependent calculations for the reflectance $R$, transmittance $T$ and absorptance $A$ at fixed $600 \mathrm{~nm}$ wavelength. The multilayer structure consists of an incoming medium of F2-glass $\left(n_{0}=1.67\right)$, a silica layer $\left(n_{1}=1.46 ; \mathrm{d}_{1}=400 \mathrm{~nm}\right)$, a light absorbing layer $\left(\tilde{n}_{2}=1.8-0.02 i ; \mathrm{d}_{2}\right)$ and an outgoing medium of silica $\left(n_{3}=1.46\right)$. The simulations for the two thicknesses are given in the Figures 4 and 5. The case with thin absorptive layer serves to demonstrate a numerical inaccuracy of the ill-conditioned model (i.e. inaccurate results), whereas the case with thick absorptive layer demonstrates a numerical singular instability of the ill-conditioned model (i.e. singular results), at certain critical conditions.

The first simulations are given for a thin absorbing layer with $\tilde{n}_{2}=1.8-0.02 i ; \mathrm{d}_{2}=10 \mathrm{~nm}$ (see Fig. 4). It can be observed that the (3)-(4)-(5) model yields $R+T+A>1$ and $R>1 ; T>1 ; A<1$, which are physically invalid, indicating inaccuracies in the numerical calculation. The origin of these inaccuracies is nevertheless not the exponential instabilities discussed in detail in thin paper, but a bad matrix factorization of the TMM that is enhanced at the critical angle $\theta_{T I R}=\arcsin \left[n_{1} / n_{0}\right] \approx 61^{\circ}$ (see Fig. 4). We can see this and classify it as a round off error by investigating the numerical values in the sub-matrices of the illconditioned case, as follows. First, note that the transfer matrix for the well-conditioned model (5)-(9)-(10) 
in the case of this simple multilayer is represented by $\mathbf{M}=\mathbf{D}_{3}^{-1} \mathbf{D}_{2} \mathbf{P}_{2}^{-1} \mathbf{D}_{2}^{-1} \mathbf{D}_{1} \mathbf{P}_{1}^{-1} \mathbf{D}_{1}^{-1} \mathbf{D}_{0}$. The same multilayer is represented in the ill-conditioned model (3)-(4)-(5) with the same transfer matrix, but with the propagation matrices defined slightly differently due to the change of the reference system. Using above matrix notation of the well-conditioned model, the transfer matrix of the ill-conditioned model can thus be written as $\mathbf{M}=\left[\left(\mathbf{P}_{3}\right)_{\left(y_{2}-y_{0}\right)} \mathbf{D}_{3}^{-1}\right]\left[\mathbf{D}_{2}\left(\mathbf{P}_{2}\right)_{\left(y_{2}-y_{1}\right)}^{-1} \mathbf{D}_{2}^{-1}\right]\left[\mathbf{D}_{1}\left(\mathbf{P}_{1}\right)_{\left(y_{1}-y_{0}\right)}^{-1} \mathbf{D}_{1}^{-1}\right]\left[\mathbf{D}_{0}\left(\mathbf{P}_{0}\right)_{\left(y_{0}-y_{0}\right)}^{-1}\right]$. In addition to this, in order to access the numerical values of the sub-matrices corresponding to each layer (the squared parenthesis in the transfer matrix), we have to pay attention to the normalization that is implicit in the TMM via the continuity boundary conditions of the Maxwell's equations. As a result of these boundary conditions (well known, and thus not shown here explicitly), the second row in the sub-matrices becomes the derivative in position of the first row (more precisely the derivative divided by the magnetic permeability for the $s$ polarization, and the derivative divided by the electric permittivity for the $p$-polarization in the corresponding layer). We denote this by $\mathbf{M}=\left[\left(\mathbf{P}_{3}\right)_{\left(y_{2}-y_{0}\right)} \mathbf{D}_{3}^{-1}\right]^{R}\left[\mathbf{D}_{2}\left(\mathbf{P}_{2}\right)_{\left(y_{2}-y_{1}\right)}^{-1} \mathbf{D}_{2}^{-1}\right]^{R}\left[\mathbf{D}_{1}\left(\mathbf{P}_{1}\right)_{\left(y_{1}-y_{0}\right)}^{-1} \mathbf{D}_{1}^{-1}\right]^{R}\left[\mathbf{D}_{0}\left(\mathbf{P}_{0}\right)_{\left(y_{0}-y_{0}\right)}^{-1}\right]^{R}$. This normalization recovers the original structure of the transfer matrix taking into account the continuity boundary conditions of the Maxwell's equations without any other additional change. It turns out that in the presented case, the ill-conditioned model showed numerical inaccuracy (Fig.4b) originating from two of the the matrix groups, namely $\left[\mathbf{D}_{0}\left(\mathbf{P}_{0}\right)_{\left(y_{0}-y_{0}\right)}^{-1}\right]^{R}$, that depends on $y_{0}$, and $\left[\left(\mathbf{P}_{3}\right)_{\left(y_{2}-y_{0}\right)} \mathbf{D}_{3}^{-1}\right]^{R}$, that depends on $y_{2}=y_{0}+d$, where $d=410 \mathrm{~nm}$ is the total thickness of the multilayer and $y_{0}=0 \mathrm{~nm}$ is the origin. To show this we computed these two grouped matrices separately from the TMM for both the $s$ - and $p$-polarization modes, for the particular case of wavelength $\lambda=600 \mathrm{~nm}$ and angle $\theta_{0}=70^{\circ} \equiv 2 \times \pi \times 70 / 360 \mathrm{rad}$, and the results are shown below. 


$$
\begin{aligned}
& {\left[\mathbf{D}_{0}\left(\mathbf{P}_{0}\right)_{\left(y_{0}-y_{0}\right)}^{-1}\right]_{s}^{R}=\left(\begin{array}{cc}
e^{-i \beta_{0}\left(y_{0}-y_{0}\right)} & e^{i \beta_{0}\left(y_{0}-y_{0}\right)} \\
-\frac{2 \pi n_{0} \cos \left(\theta_{0}\right)}{\lambda} e^{-i \tilde{\beta}_{0}\left(y_{0}-y_{0}\right)} & \frac{2 \pi n_{0} \cos \left(\theta_{0}\right)}{\lambda} e^{i \beta_{0}\left(y_{0}-y_{0}\right)}
\end{array}\right)=\left(\begin{array}{cc}
1 & 1 \\
-6.0 \times 10^{6} & 6.0 \times 10^{6}
\end{array}\right)}
\end{aligned}
$$

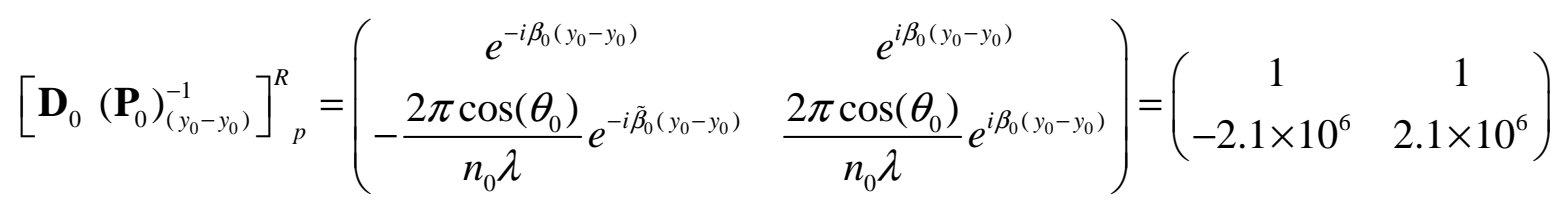

$$
\begin{aligned}
& \left(\left[\mathbf{D}_{3}\left(\mathbf{P}_{3}\right)_{\left(y_{2}-y_{0}\right)}^{-1}\right]^{-1}\right)_{s}^{R}=\left[\left(\mathbf{P}_{3}\right)_{\left(y_{2}-y_{0}\right)} \mathbf{D}_{3}^{-1}\right]_{s}^{R}=\left(\begin{array}{ll}
\frac{1}{2} e^{i \tilde{\beta}_{3}\left(y_{0}+d\right)} & -\frac{\lambda}{4 \pi n_{3} \cos \left(\tilde{\theta}_{3}\right)} e^{i \tilde{\beta}_{3}\left(y_{0}+d\right)} \\
\frac{1}{2} e^{-i \tilde{\beta}_{3}\left(y_{0}+d\right)} & \frac{\lambda}{4 \pi n_{3} \cos \left(\tilde{\theta}_{3}\right)} e^{-i \tilde{\beta}_{3}\left(y_{0}+d\right)}
\end{array}\right)= \\
& =\left(\begin{array}{cc}
0.0423-0.0000 i & -1.6 \times 10^{-13}-1.1 \times 10^{-8} i \\
5.9117+0.0001 i & -5.6 \times 10^{-11}+1.6 \times 10^{-6} i
\end{array}\right) \\
& \left(\left[\mathbf{D}_{3}\left(\mathbf{P}_{3}\right)_{\left(y_{2}-y_{0}\right)}^{-1}\right]^{-1}\right)_{p}^{R}=\left[\left(\mathbf{P}_{3}\right)_{\left(y_{2}-y_{0}\right)} \mathbf{D}_{3}^{-1}\right]_{p}^{R}=\left(\begin{array}{ll}
\frac{1}{2} e^{i \tilde{\beta}_{3}\left(y_{0}+d\right)} & -\frac{\lambda n_{3}}{4 \pi \cos \left(\tilde{\theta}_{3}\right)} e^{i \tilde{\beta}_{3}\left(y_{0}+d\right)} \\
\frac{1}{2} e^{-i \tilde{\beta}_{3}\left(y_{0}+d\right)} & \frac{\lambda n_{3}}{4 \pi \cos \left(\tilde{\theta}_{3}\right)} e^{-i \tilde{\beta}_{3}\left(y_{0}+d\right)}
\end{array}\right)= \\
& =\left(\begin{array}{cc}
0.0423-0.0000 i & 2.6 \times 10^{-13}+7.5 \times 10^{-9} i \\
5.9117+0.0001 i & 1.5 \times 10^{-11}-1.0 \times 10^{-6} i
\end{array}\right)
\end{aligned}
$$

The calculations shown in (11) and (12) suggest that the origin for the inaccuracies in this case is a round off due to a large difference in the order of magnitude between the different components of a same matrix. As can be seen from (11) and (12) the orders of magnitude difference in the matrix components comes from very high $1 / \lambda$ and very low $\lambda$ factors multiplying the exponentials. This origin of this inaccuracy is thus different than the numerical instability associated to large exponentials in the propagation matrices, discussed in Section 4, which is understandable since in this example, the one absorptive layer in the multilayer was not very thick, unlike in the second example discussed below. Nevertheless, it turns out that the normalization used to obtain the well-conditioned model removes this inaccuracy related to the selective matrix element scaling by $1 / \lambda$ and $\lambda$, since the well-conditioned model consider a matrix factorization where these scales are exactly compensated. 
The second simulation example demonstrates the numerical instabilities related the large exponentials and their prevention by the well-conditioned model, which is main topic of the paper. Simulation results are shown for a thick absorbing layer with $\tilde{n}_{2}=1.8-0.02 i ; \mathrm{d}_{2}=1 \mathrm{~mm}$ (see Fig. 5). It can be seen that the (3)(4)-(5) model yields $R+T+A>>1$ and $R>>1$; $T>>1$; $A<<1$, indicating now even higher inaccuracy (round off) than in the previous case (compare the Fig. 5b with the Fig. 4b). Furthermore, the round off error turns now even to an overflow with an exponential origin due to the a combination of two different exponential effects: scale accumulation through the imaginary part of the refractive index due to a strongly absorbing layer in one hand, and scale accumulation due to an imaginary part in the angle due to TIR on the other. Therefore, the overflow arises just at angles higher than the TIR critical angle $\theta \geq \theta_{T I R} \approx 61^{\circ}$ (see Fig. $5)$.

The simulations for both thicknesses with the (5)-(9)-(10) model give well-conditioned results (Fig. 4a, Fig. 5a) which arises from the fact that this model takes into account the internal symmetries of the system (homogeneity by domain). Therefore, the physical homogeneity of the interaction system, which appears explicitly as internal symmetries in the mathematical structure of the TMM, represents the advantage that it not only simplifies the model analytically, as we showed in the theoretical sections, but also improves its numerical conditioning, as we have illustrated in this section with the practical simulations.

The current paper developed a new general symmetry formalism that improves the numerical accuracy and stability of non-periodic 1-dimensional systems. The new formalism used 1-dimensional translations and dilatations that were possible due to the layer-wise homogenous and isotropic properties of the multilayer system inherently assumed in the transfer matrix method. Although such modifications to the TMM are well known and adopted in practice [12], we believe that the symmetry perspective taken here is fruitful for understanding, handling and comparing different analytical modifications to the TMM formalism. As a final remark, we point out that since symmetry theory and differential geometry are in general powerful tools for understanding and solving also higher-dimensional problems in solid state physics (optics, mechanics, etc), it is interesting to speculate, and to propose as a topic for future work, whether the ideas presented here could be extended also to higher dimensional systems and thus help understand and manage their numerical 
instabilities, by introducing adequate 2- and 3-dimensional transformations adapted to the geometries of those systems.

\section{Conclusions}

In this paper we have analytically and numerically characterized the effect of the translational-dilatational internal symmetries (homogeneity by domain) in the structure of the transfer matrix method (TMM) for a one-dimensional multilayer. These internal symmetries allow using reference system transformations as an efficient tool to simplify the TMM and improve its numerical behaviour. The theoretical development was based on recognizing both: $a$ ) the internal symmetry of the interaction system arising from the homogeneity by domain, and $b$ ) transformation of reference system towards an improved TMM mapping. These theoretical conclusions were illustrated by simulations of specific systems showing numerical inaccuracies and instabilities under the conditions of total internal reflection (TIR) and/or high optically thick absorptive layers, due to scale accumulation through the exponential terms of the TMM.

In away, this is a geometrical method as it employs transformations of the reference system in such a way that they are adequately fitted to the internal symmetries of the interaction system. Therefore, we propose the symmetry method as a potentially powerful procedure to analyze and resolve different analytical and numerical problems which could appear in the resolution of optical systems at different conditions.

\section{Acknowledgements}

This work was partially funded by the Nordic Innovation Center project NORDIC DSC (no. 09053) and the CNB-E project of the TKK/Aalto University Multidisciplinary Institute of Digitalisation and Energy MIDE. H.M. thanks the Spanish Ministry of Economy and Competitiveness for funding under grants MAT2011-23593 and Consolider CSD2007-00007 and Junta de Andalucia for funding under grants FQM3579 and FQM-5247. H.M. also thanks the European Research Council for a Starting Grant (POLIGHT). 


\section{References}

[1] M. G. Moharam, D. A. Pommet, E. B. Grann, and T. K. Gaylord, Stable implementation of the rigorous coupled-wave analysis for surface-relief gratings: enhanced transmittance matrix approach, J. Opt. Soc. Am. A 12,1077-1086 (1995)

[2] L. Li, Formulation and comparison of two recursive matrix algorithms for modeling layered diffraction gratings, J. Opt. Soc. Am. A 13, 1024-1035 (1996)

[3] M. Skorobogatiy and J.Yang, Fundamentals of Photonic Crystal Guiding. Cambridge: Cambridge University Press, 2009

[4] R. L. Burden and J. D. Faires, Numerical Analysis. Boston: Brooks Cole, 2010, chap. 1

[5] D. Maystre, S. Enoch, and G. Tayeb, Scattering Matrix Method applied to photonic crystals, in Electromagnetic Theory and Applications for Photonic Crystals, K. Yasumoto (ed.), Boca Raton FL: Taylor \& Francis, 2006, chap. 1, pp. 1-45

[6] D. Y. K. Ko and J. R. Sambles, Scattering Matrix Method for propagation of radiation in stratified media: attenuated total reflection studies of liquid crystals, J. Opt. Soc. Am. A 5,1863-1866 (1988)

[7] S. A. Dyakov, V. A. Tolmachev, E. V. Astrova, S. G. Tikhodeev, V. Y. Timoshenko and T. S. Perova, Numerical methods for calculation of optical properties of layered structures, International Conference on Micro- and Nano-Electronics 2009, Proc. of SPIE, Vol. 7521, 75210G.

[8] L. C. Botten, N. A. Nicorovici, R. C. McPhedran, C. Martijn de Sterke and A. A. Asatryan, Photonic band structure calculations using scattering matrices, Phys. Rev. E 64, 046603 (2001)

[9] B. Gralak, M. de Dood, G. Tayeb, S. Enoch and D. Maystre, Theoretical study of photonic band gaps in woodpile crystals, Phys. Rev. E 67, 066601 (2003)

[10] B. Gralak, S. Enoch and G. Tayeb, From scattering or impedance matrices to Bloch modes of photonic crystals, J. Opt. Soc. Am. A 19, 1547 (2002) 
[11] Q. Cao, P. Lalanne and J.-P. Hugonin, Stable and efficient Bloch-mode computational method for onedimensional grating waveguides, J. Opt. Soc. Am. A 19, 335 (2002)

[12] P. Yeh, Optical Waves in Layered Media. Hoboken: Wiley-Interscience, 2005, chaps. 5 and 6 


\section{Figure Captions}

Figure 1. Structure of the multilayer system with its electromagnetic and geometrical properties, where $\tilde{\varepsilon}_{j}, \tilde{\mu}_{j}, \tilde{n}_{j}$ are the complex electric permittivity, complex magnetic permeability and the complex refractive index, respectively, for the layer $j=1, \ldots, n$ or external media $j=0, n+1$. The thickness of each layer are characterized by $d_{j}$ with $j=1, \ldots, n$, and the interfaces by $y_{j}$ with $j=0, \ldots, n$.

Figure 2. Vectorial electromagnetic propagation in one layer or external media $j$ from the multilayer system, where $j=0,1, \ldots, n, n+1$. The propagation is split in two modes: the transversal electric (TE) or $s$ polarization (on the left) and the transversal magnetic (TM) or p-polarization (on the right). The propagation of each mode contains both forward and backward directions (this last not marked in the figure); furthermore, each propagation direction contains the multiple reflections generated in the other interfaces of the multilayer.

Figure 3. Graphical representation of the multilayer (colored area) showing a transformation of reference system (7) and its mapping between propagation matrices (8) that consider the internal symmetries of the TMM (that is, it considers each domain as homogeneous and isotropic). The dynamical matrices (not showed) that are associated with the interface points, are invariant in these transformations. The initial reference system $\left\{y ; y_{0}\right\}$ corresponds to the solution (3)-(4)-(5), while the final domain-wise reference system $\left\{\left(y ; y_{0}\right) \cup\left(y ; y_{1}-y_{0}\right) \cup \ldots \cup\left(y ; y_{n}-y_{n-1}\right)\right\}$ corresponds to the solution (5)-(9)-(10). The reference systems are indicated as follow: the interface points by blue notation, the domains with bold dark italic notation and the origins are marked by hollow circles. 
Figure 4. Calculation at $600 \mathrm{~nm}$ wavelength for total internal reflection (TIR) with a very thin absorptive layer, practically without absorption. The reflectance, transmittance and absorptance are compared between a) the improved (well-conditioned) model given by (5)-(9)-(10), and $b$ ) the conventional (ill-conditioned) model given by (3)-(4)-(5). It can be seen that the ill-conditioned calculation produces the inaccuracies $R+T+A>1$ and $R>1 ; T>1 ; A<1$ due to the TIR at angles higher than 60 , while the well-conditioned calculation yields correct results. The behaviour at other visible wavelengths is similar (not shown).

Figure 5. Same calculations as in Figure 4, but with thicker absorptive layer. It can be seen that with the illconditioned calculation the numerical inaccuracies are now more pronounced, due to the high thickness. Numerical overflow appears at the critical incident angle and higher $\theta \geq \theta_{T I R} \approx 61^{\circ}$, due to contributions from both TIR and strong absorption, while the well-conditioned calculation yields again correct results. Also in this case the behaviour at other visible wavelengths is similar (not shown). 


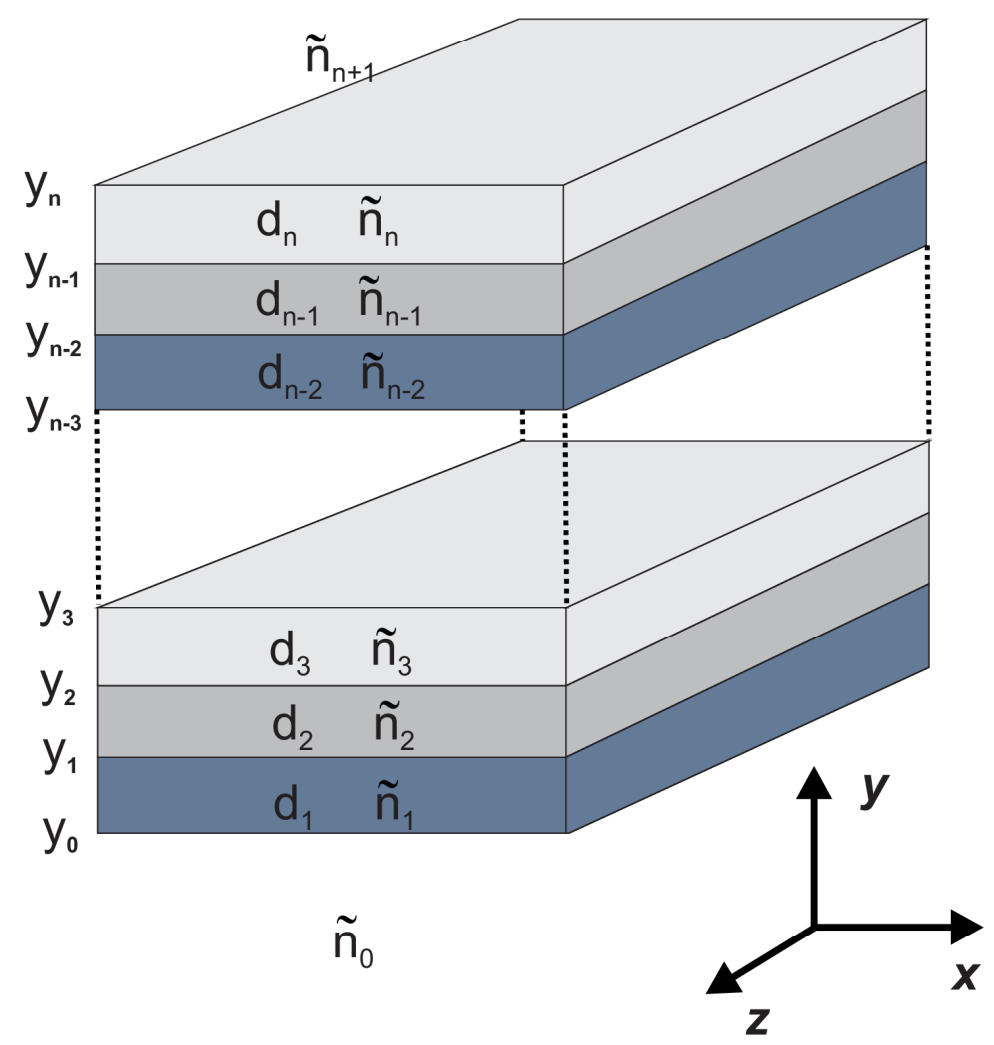

Figure 1. Structure of the multilayer system with its electromagnetic and geometrical properties, where $\tilde{\varepsilon}_{j}, \tilde{\mu}_{j}, \tilde{n}_{j}$ are the complex electric permittivity, complex magnetic permeability and the complex refractive index, respectively, for the layer $j=1, \ldots, n$ or external media $j=0, n+1$. The thickness of each layer are characterized by $d_{j}$ with $j=1, \ldots, n$, and the interfaces by $y_{j}$ with $j=0, \ldots, n$. 


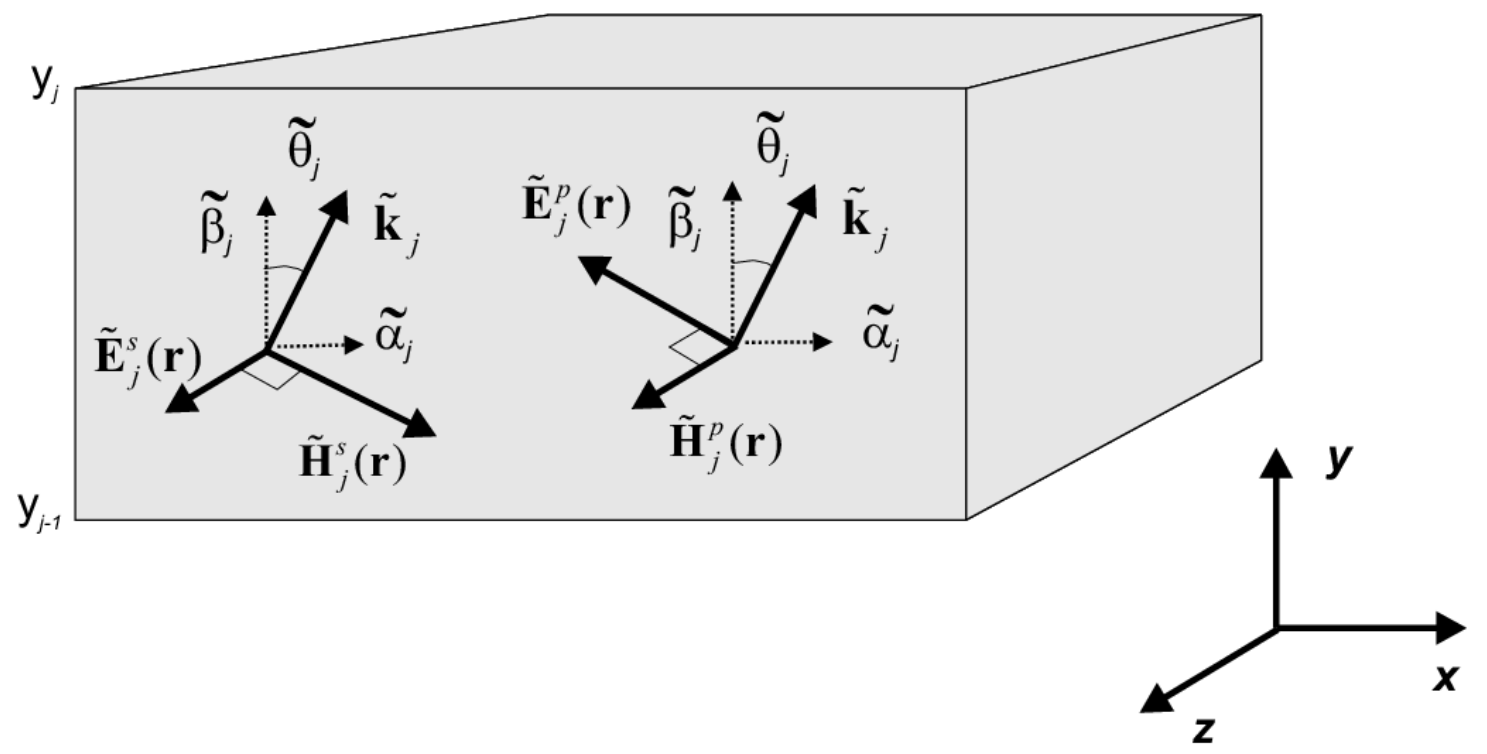

Figure 2. Vectorial electromagnetic propagation in one layer or external media $j$ from the multilayer system, where $j=0,1, \ldots, n, n+1$. The propagation is split in two modes: the transversal electric (TE) or $s$ polarization (on the left) and the transversal magnetic (TM) or p-polarization (on the right). The propagation of each mode contains both forward and backward directions (this last not marked in the figure); furthermore, each propagation direction contains the multiple reflections generated in the other interfaces of the multilayer. 


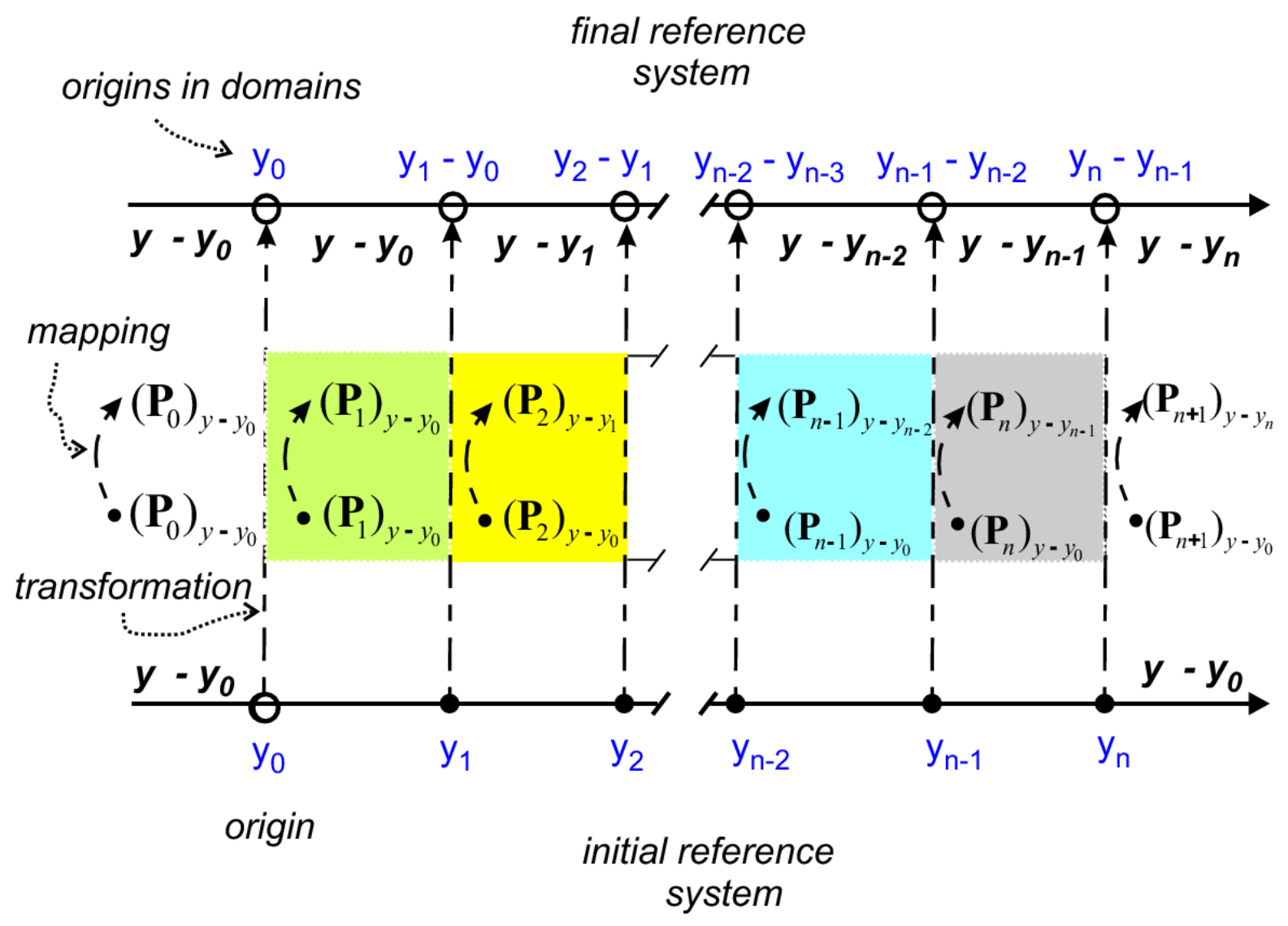

Figure 3. Graphical representation of the multilayer (colored area) showing a transformation of reference system (7) and its mapping between propagation matrices (8) that consider the internal symmetries of the TMM (that is, it considers each domain as homogeneous and isotropic). The dynamical matrices (not showed) that are associated with the interface points, are invariant in these transformations. The initial reference system $\left\{y ; y_{0}\right\}$ corresponds to the solution (3)-(4)-(5), while the final domain-wise reference system $\left\{\left(y ; y_{0}\right) \cup\left(y ; y_{1}-y_{0}\right) \cup \ldots \cup\left(y ; y_{n}-y_{n-1}\right)\right\}$ corresponds to the solution (5)-(9)-(10). The reference systems are indicated as follow: the interface points by blue notation, the domains with bold dark italic notation and the origins are marked by hollow circles. 
a)
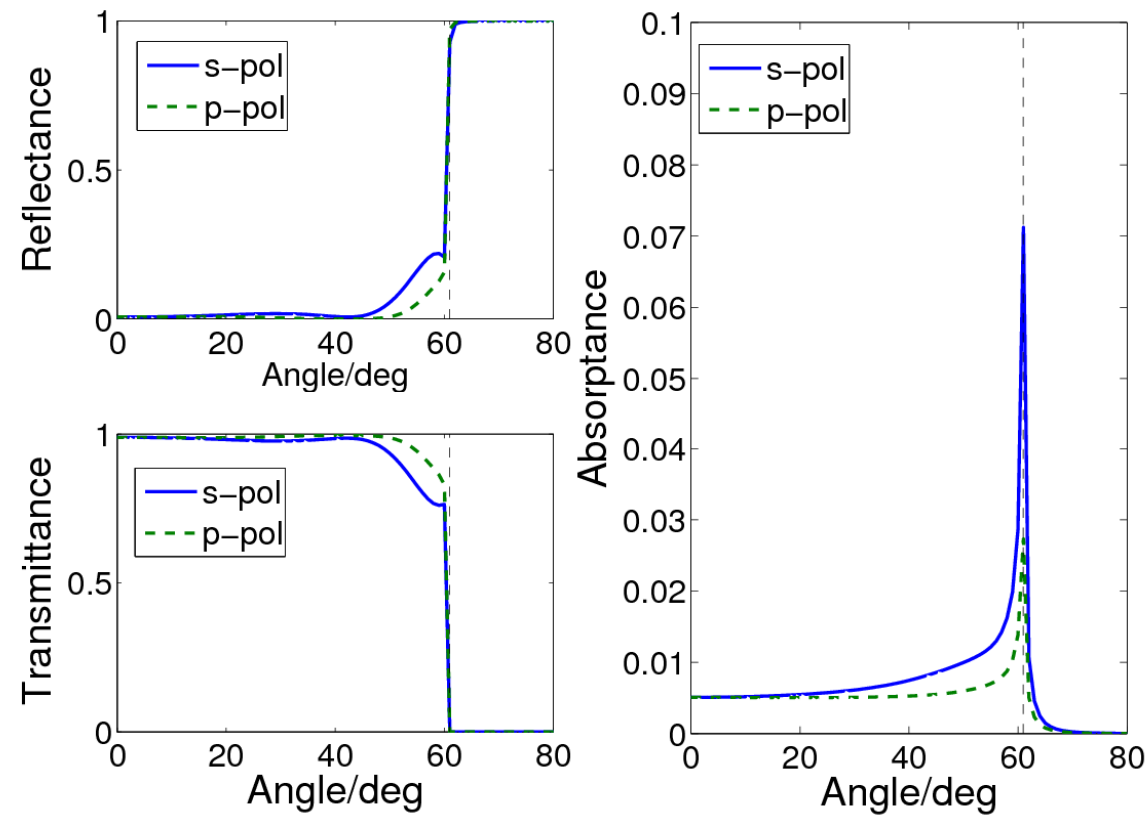

b)
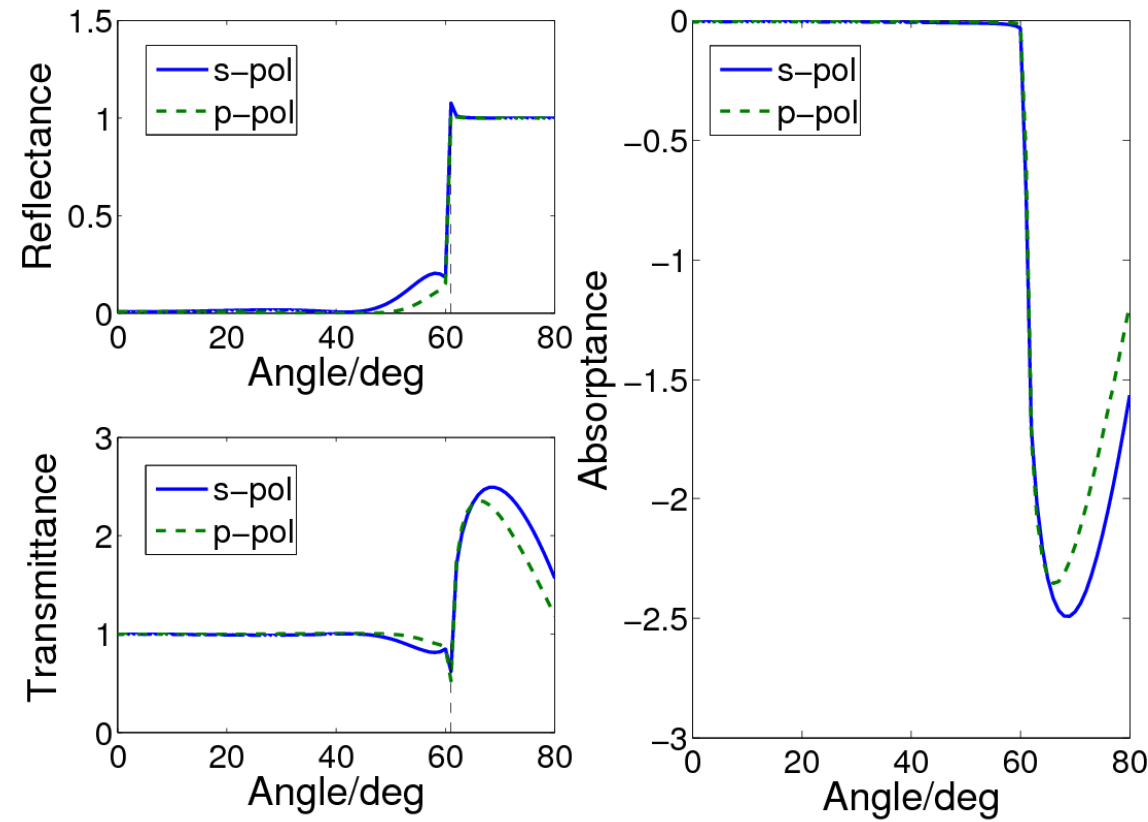

Figure 4. Calculation at $600 \mathrm{~nm}$ wavelength for total internal reflection (TIR) with a very thin absorptive layer, practically without absorption. The reflectance, transmittance and absorptance are compared between a) the improved (well-conditioned) model given by (5)-(9)-(10), and $b$ ) the conventional (ill-conditioned) model given by (3)-(4)-(5). It can be seen that the ill-conditioned calculation produces the inaccuracies $R+T+A>1$ and $R>1 ; T>1 ; A<1$ due to the TIR at angles higher than 60 , while the well-conditioned calculation yields correct results. The behaviour at other visible wavelengths is similar (not shown). 
a)
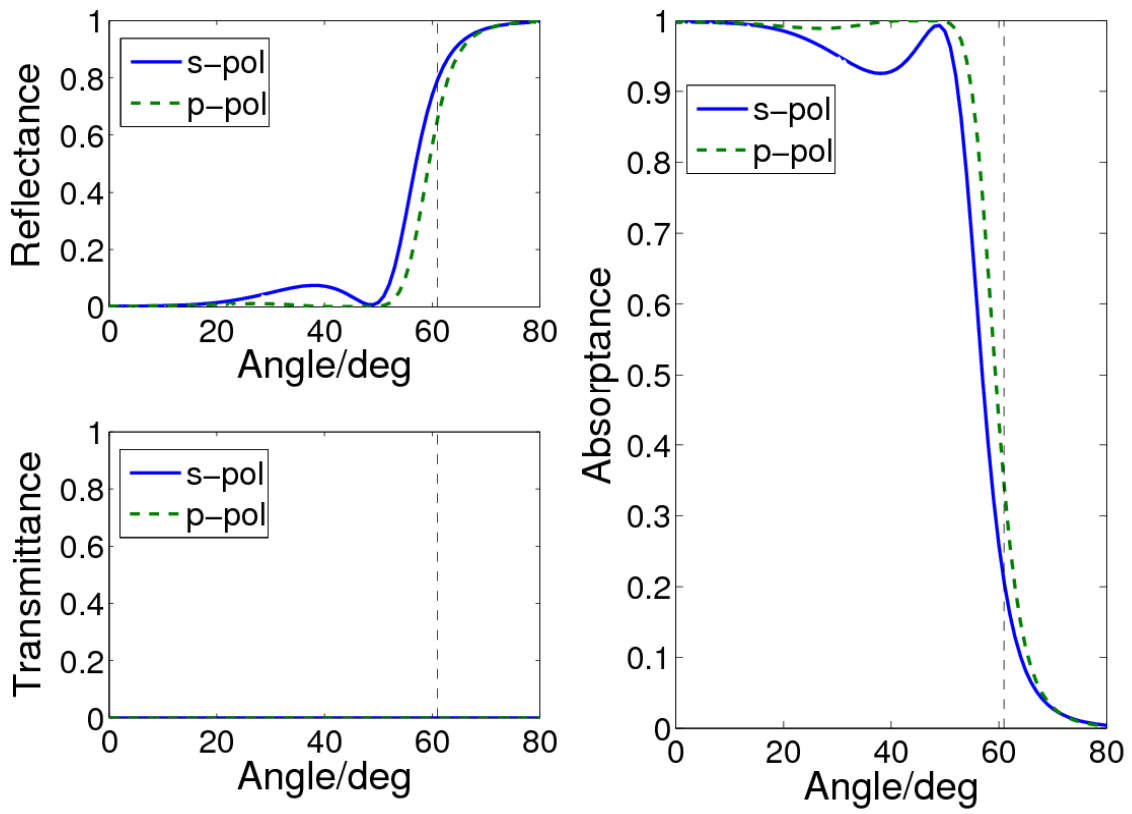

b)
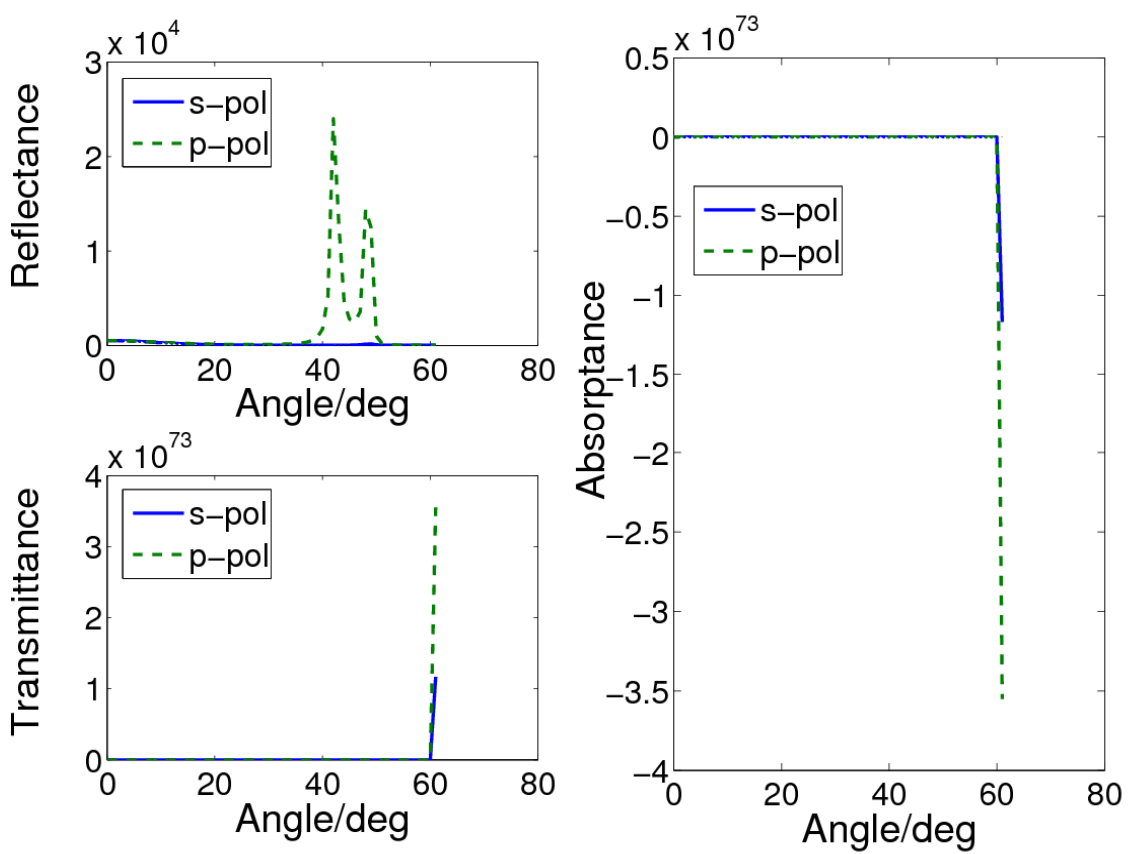

Figure 5. Same calculations as in Figure 4, but with thicker absorptive layer. It can be seen that with the illconditioned calculation the numerical inaccuracies are now more pronounced, due to the high thickness. Numerical overflow appears at the critical incident angle and higher $\theta \geq \theta_{T I R} \approx 61^{\circ}$, due to contributions from both TIR and strong absorption, while the well-conditioned calculation yields again correct results. Also in this case the behaviour at other visible wavelengths is similar (not shown). 